\title{
Abstract Methods on Mesoscopic Scales of Friction
}

\author{
Georg-Peter Ostermeyer and Andreas Krumm
}

\begin{abstract}
In recent years, research has increasingly focused on the complex processes involved in friction contacts. Especially in tribological high-loaded contacts, characterized by the presence of contact modifying wear particles, macroscopic friction shows a surprisingly high dynamic complexity on many temporal and local scales. There are dominant effects on mesoscopic scales such as the geometric self-organization structures of the wear dust in the contact, which can significantly change the local contact surfaces. For the description and simulation of these phenomena, abstract methods have shown their effectiveness. One class of methods are cellular automata, both volume- and particle-based. The latter are in particular the Movable Cellular Automata developed by Sergey Psakhie. The scales of these discrete methods are freely selectable in wide ranges between the macro world and the atomic scale. Nevertheless, they provide reliable information on mesoscopic balances in the boundary layer and thus also on the macroscopic behavior of the tribocontact. The success of these methods is shown by the example of an automotive brake. The question of the relative insensitivity of the scales of these mesoscopic methods is examined in detail.
\end{abstract}

Keywords Dynamic friction - Dynamic equilibrium $\cdot$ Mesoscopic scales $\cdot$ Cellular automata $\cdot$ Particle methods $\cdot$ Dissipation of information

\section{Introduction}

Normally, the introduction to works on friction provides a quick overview or excerpt from 5000 years of history on friction. Starting with the fire generated by friction in the Stone Age to modern theories of friction. Although friction is omnipresent, it is still far from being understood well.

\footnotetext{
G.-P. Ostermeyer $(\varangle) \cdot$ A. Krumm

Institut für Dynamik und Schwingungen, Technische Universität Braunschweig, Schleinitzstraße 20, 38106 Braunschweig, Germany

e-mail: gp.ostermeyer@tu-braunschweig.de
} 
This paper provides not a historical but an "engineering" view to friction as a "friction machine". Imagine you have to design a machine that generates the following:

- forces,

- heat,

- vibration,

- noise,

- particles and dust

and in addition includes:

- surface roughening,

- surface smoothing,

- welding processes,

- modifications of the crystalline surface structure and

- chemical oxidation processes.

It is very simple to realize this kind of machine: Two solid bodies in contact, which are shifted tangentially against each other, can show all the phenomena mentioned above (see Fig. 1).

The paradox between the geometric simplicity of the machine and the complexity of the physical phenomena observed is the reason for the difficulty in describing friction comprehensively. Only in very few cases is it possible to adjust some of the processes mentioned above a priori by designing this "friction machine" or "boundary layer machine" [1].

It is interesting that people have frequently tried to describe these machines with a single scalar quantity $\mu$ [2]. This quantity is defined as the quotient of frictional and normal force.

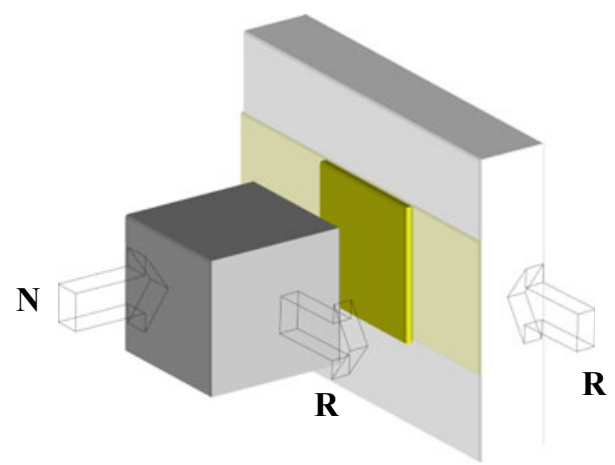

Fig. 1 Design of a friction machine 


$$
\mu=\frac{\|\vec{R}\|}{\|\vec{N}\|}
$$

Measurements repeatedly confirmed that the coefficient of sliding friction depends on the speed, surface roughness, the materials involved, the ambient medium and many other parameters. It may even depend on quantities that can be explained by the measuring principle of the friction force rather than by the physics of friction. Recent investigations suggest that this coefficient is dynamic, i.e. it is only indistinctly represented by stationary measurements [3].

The following interpretation may explain why the friction coefficient is nevertheless useful. If the numerator and denominator in (1) are multiplied by the tangential velocity vector,

$$
\mu=\frac{\vec{R} \cdot \vec{v}}{\|\vec{N}\| \cdot\|\vec{v}\|}
$$

the friction coefficient can be interpreted as friction power related to a characteristic system performance [1]. Power is certainly a good measure to describe the effect of a machine. With this interpretation energetic connections to the above mentioned phenomena of a friction machine can be presented and explained. This applies in particular to heat regeneration or the averaged wear volume.

If you want to take a closer look at the physics of the machine, you have to move from the symbolic point contact (1) to the consideration of the area of the frictional contact. This is connected with a change of scale of the considered lengths and times. The scales are very different for the processes of the boundary layer machine mentioned above. They often lie between the macroscopic world of experience and the atomic scale. Consideration of mesoscopic scales have proven to be very useful for establishing connections both with macro and micro scales. To approach the meso scale, one can start from the atomic scale ("Bottom Up") or the macroscopic scale ("Top Down").

\section{Bottom-Up View}

On the atomic scale, with a characteristic length of $10^{-10} \mathrm{~m}$, energy distribution processes can be traced by phonons and atomic movements. Today's non-equilibrium molecular dynamics programs are able to describe atomic or molecular assemblies in a thermodynamically and quantum-mechanically correct way. In principle, this method yields approximately $10^{25}$ equations per $\mathrm{cm}^{3}$ of matter. The time constants of molecular dynamics are on the order of femtoseconds. With today's computers, therefore, no general statements about friction on the macroscopic scale are possible. 
The transition from the atomic scale to larger length scales is combined with a drastic reduction in the number of descriptive equations of motion. Classical mechanics offers a perspective on this matter. There are different ways to transfer atomic information into the macroscopic world.

On the one hand, atomic or molecular assemblies can be made ever larger by reducing the number of particles but increasing their mass. This results in an ensemble of particles which are the core of the so-called Discrete Element Methods (DEM). If these atomic substitutes are further coarsened, only a few rigid bodies may remain. These are objects of the Multibody Systems Methods (MBS).

Another very successful way of mechanics to transform information from the atomic level to the macroscopic world is the smoothing out of the atomic structure to a massy continuum. Like the other two methods, this can be treated mathematically with very small effort. Numerical methods like finite element methods or boundary element methods (FEM, BEM) are being used.

In this way, mass-equivalent models can be created that are easy to handle mathematically. Interestingly, the force influences that are essential for the equations of motion are determined by measurement from the macroscopic world of experience alone and integrated into the models. Any phenomena for the material or motion behaviour can be approximated with arbitrary accuracy using a few generic force elements. This works quite perfectly in may situations, but fails completely in the case of friction forces.

One method for a better description of friction could be to transfer force information from the atomic scale to the macroscopic experiential using the mesoscopic particle method $[4,5]$.

In fact, as early as 1890 , Boltzmann made the first proposals to connect the microscopic particle world with the macroscopic mechanical world [6]. Pioneering papers on this subject have been published by Greenspan [7, 8]. DEM provides excellent results for macroscopic motion and deformation dynamics, but it shows its weakness in thermodynamics. Although the thermodynamic processes can be calculated well with the MD simulation by statistical and stochastic methods, these methods cannot describe dynamic effects in the macroscopic world due to their time constants.

The approach of mesoscopic particles is to apply an atomic substitute world on a mesoscopic scale, which is below but close to the macroscopic scale (see Fig. 2). This mesoscopic world makes it possible to couple the particles to the mechanical bodies by "hidden" degrees of freedom. These additional degrees of freedom allow a correct thermodynamic description of mechanical systems even with particle worlds.

The idea is to describe the frictional system on the macroscopic time scale, but the friction boundary layer on the microscopic scale. The dynamics on the microscopic scale are hidden for the macroscopic scale, just as the atomic dynamics are hidden for the macroscopic world. It only serves to detect macroscopically observable phenomena, for example heat or wear. Thus, it is possible to describe the friction process and the associated phenomena (vibrations, heat, wear, etc.) with the mesoscopic particles with arbitrarily adjustable accuracy (see Fig. 3). 


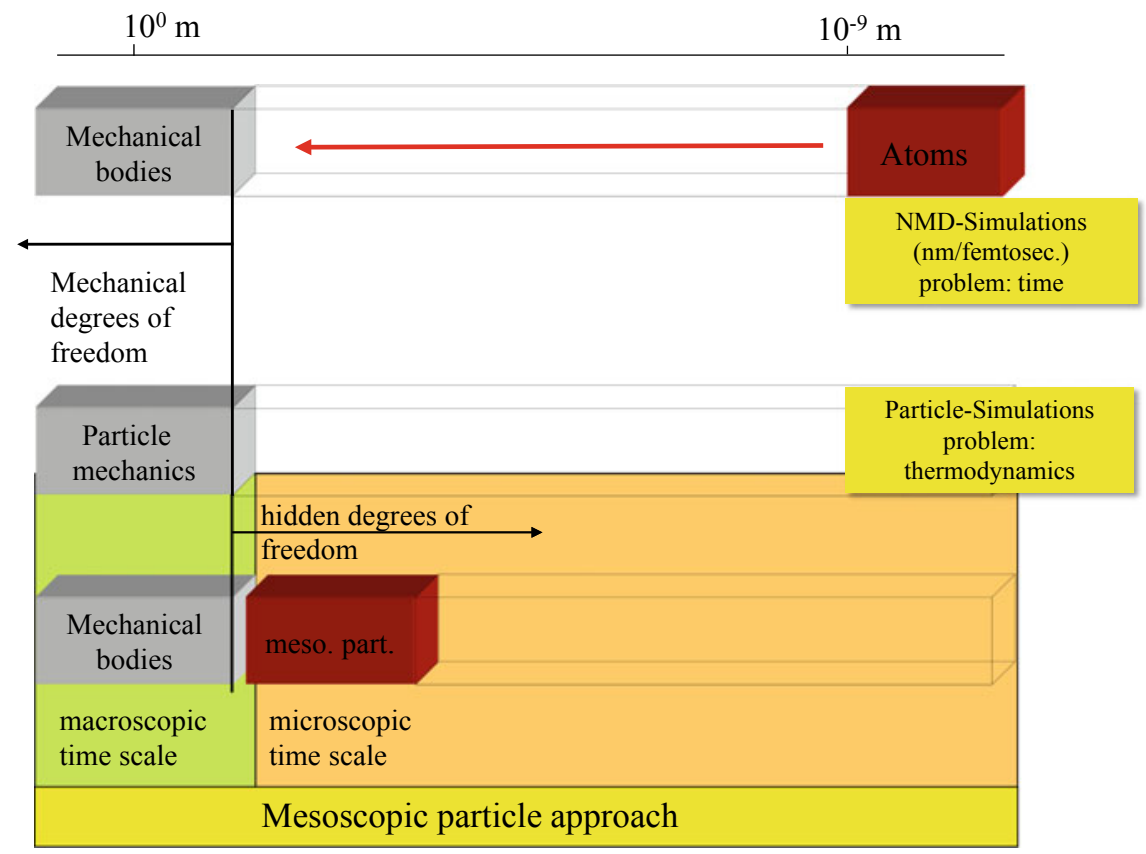

Fig. 2 Classification of the mesoscopic scale

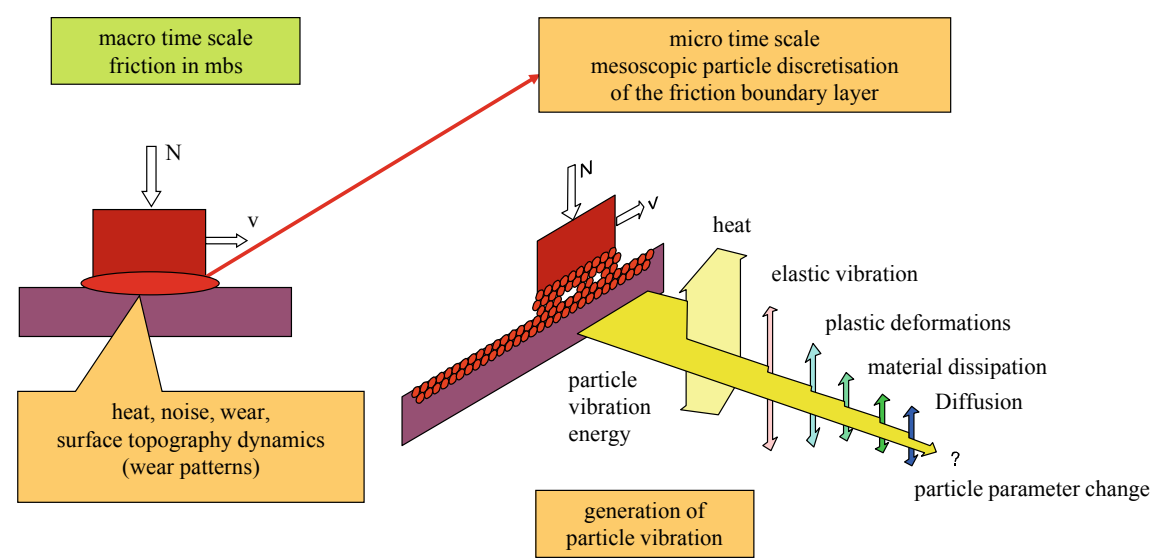

Fig. 3 Combination of macroscopic and microscopic scales in a friction system

To illustrate how this method works, a simple rod as in Fig. $4 \mathrm{a}$ is considered. If a hammer beats on the rod, the rod heats up. In this thought experiment the mechanical degrees of freedom of the rod are not considered, the elastic waves in the rod are not important. Therefore, the rod is only modelled with a sub-mechanical degree of 


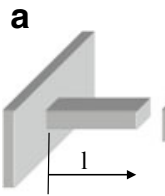

$\mathrm{m}$ b

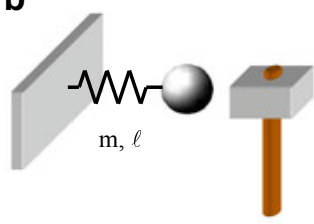

C

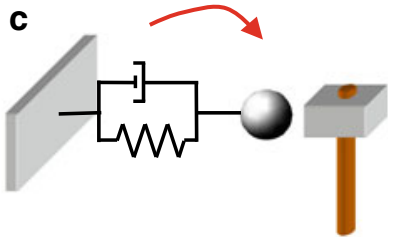

Fig. 4 a Heating a rod; b particle discretisation; $\mathbf{c}$ mesoscopic particle discretization [9]

freedom to detect heat in the rod. This sub-mechanical degree of freedom can be represented by a simple spring-mass system (see Fig. 4b).

Beaten with a hammer, the mass starts to vibrate. The system has absorbed energy from this impact. This energy can be interpreted as heat energy, the vibration itself is macroscopically irrelevant. If a second beat is given to this system, it usually leads to a violation of the thermodynamic laws, because with the second beat, the system depends essentially on the point in time at which the beat occurs, that is in which phase relation the beat and the already existing vibration stands. Thus, the vibration can be completely eliminated or the energy of the vibration can be doubled (see Fig. 5).

However, this means that the energy introduced by the second beat can no longer be interpreted as a heat gain. This energy should be added to the existing heat energy. This is exactly where the thermodynamic laws are violated.

If this mass point is, in addition to its mass property, given an internal dynamic variable, the temperature, then ultimately the sub-mechanical vibration dynamics induced by the beat can be transformed into heat. For this purpose, the vibration is dissipated by the damper, but the dissipated energy is stored in the inner variable T. In Fig. 6 this procedure is shown by means of the potential curve (blue) of the system. By the first beat a certain energy $\Delta \mathrm{E}$ is entered into the system (red). Through the damper integrated in the system, the energy introduced as an vibration is now stored in the system as heat and the potential curve is raised to this energy level. Then, a second beat can be introduced and the sub-mechanical vibration of the system induced by this
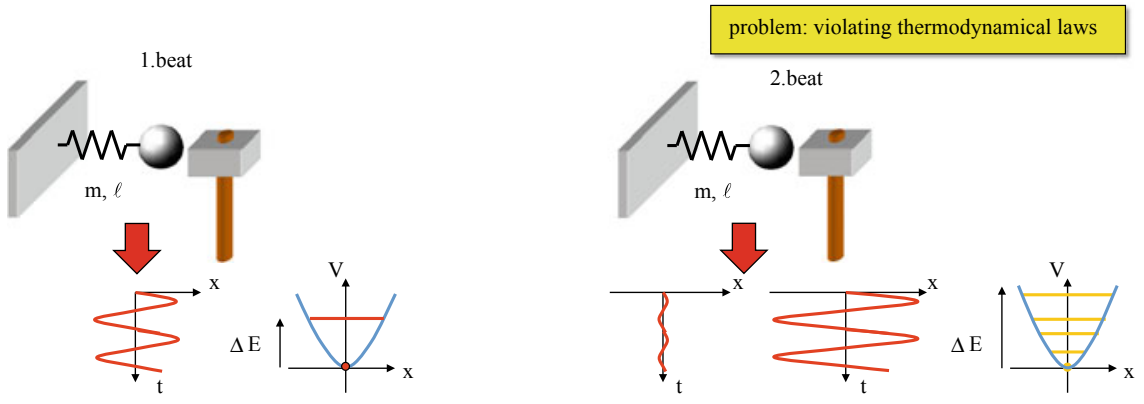

Fig. 5 Violation of the thermodynamic laws in conventional particle methods 
Time constant generate maximal frequency in macroscopic world
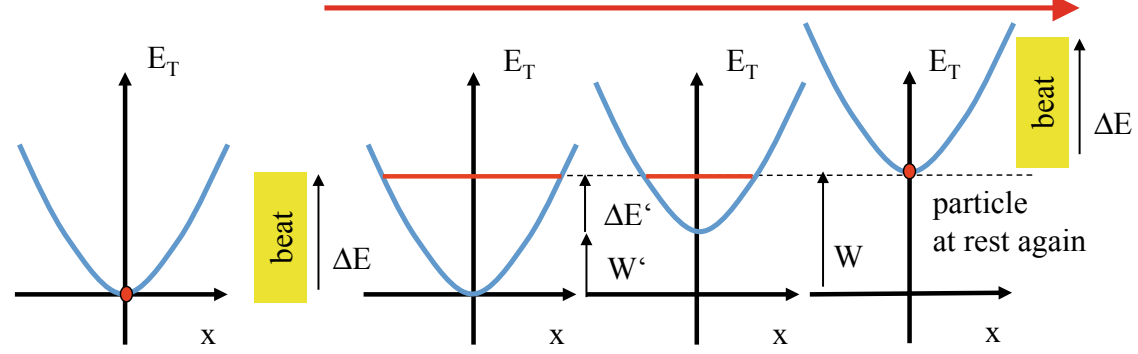

Fig. 6 Storage of thermal energy in mesoscopic particles

can be added to the existing heat energy. It should be noted that this method defines a minimum constant in terms of time, which defines the minimum distance between macroscopic impacts in order to correctly represent the thermodynamic behaviour of the system via the sub-mechanical vibrations. Such time grids can be found in all discrete systems, the grid width is a function of the typical frequencies in the grid. They limit the maximum possible frequency in the model. Every discretization method induces such grid constants. In numerical mathematics, this property is taken for granted as an essential element for the interpretation of results. However, these grid sizes can be made as small as desired by refining the sub-mechanical degrees of freedom.

The quantity of the time constant is determined by the time needed to adjust the potential curve to the correct energy level.

Mathematically this can be described by the following Lagrangian equations. The Lagrange function and the dissipation function of the system have the form

$$
L=E_{k i n}-V=\frac{1}{2} m \dot{x}^{2}-\frac{1}{2} c x^{2} \text { and } D=\frac{1}{2} b \dot{x}^{2},
$$

where $\mathrm{m}$ is the mass, $\mathrm{c}$ the stiffness of the spring, and $\mathrm{b}$ the damping coefficient.

The equations of motion are as follows:

$$
\frac{d}{d t}\left(\frac{\partial}{\partial \dot{x}} L\right)-\frac{\partial}{\partial x} L=-\frac{\partial}{\partial \dot{x}} D
$$

and the temperature change is calculated as

$$
\dot{T}=\frac{1}{m \cdot c_{v}} \dot{x} \frac{\partial}{\partial \dot{x}} D
$$

where $c_{v}$ is the specific heat capacity of the material (per unit mass) and $\mathrm{T}$ is the temperature.

The inner variable $\mathrm{T}$ gives an extended energy integral: 


$$
\frac{1}{2} m \dot{x}^{2}-\frac{1}{2} c x^{2}+m c_{v} T=\text { const } .
$$

This approach can be transferred to all possible mechanical systems. In addition to temperature, other internal variables can also be used. For example, different energy fields with different time constants are superimposed with each other. The different levels provide their macroscopically observable phenomena, and the sub-mechanical texture of the model allows the thermodynamically correct transition from one form of energy to another.

Macroscopic forces either have a zero range, for example contact or impact forces, or are described by some interaction law with a specific coordinate dependence. Forces of finite range are characteristic of atomic forces. They can be described by Lennard Jones-like potentials. In many cases, the macroscopic forces can be approximated very accurately [7].

Such Lennard-Jones potentials can also be designed temperature-dependent in such a way that transitions of the mesoscopic particles from solid to fluid or gaseous phases can be described dynamically. Figure 7 shows a metal block that is melted on a heat plate.

At the beginning of the simulation the heating plate is switched off and the particles have a defined starting temperature. After the plate is switched on, it can be observed that the temperature in the block slowly increases. As expected, the temperature of the particles in contact with the heating plate increases first. Through heat transmission, the temperature of the particles in contact with the heating plate increases. After some time it can be observed that the melting temperature of the material is reached and particles are released from the block.

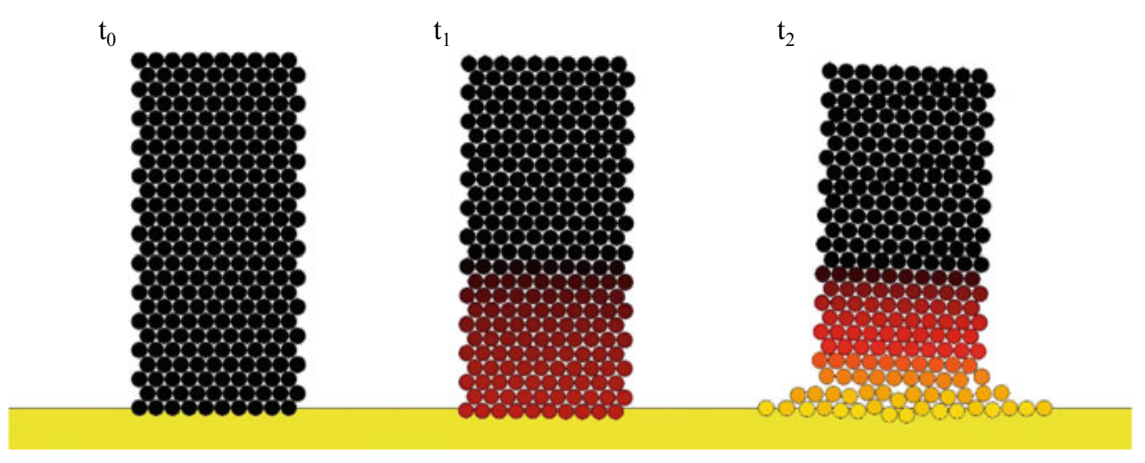

Fig. 7 A melting metal block 


\section{Top-Down View}

Friction in the macroscopic world can also be investigated more intensively using a top-down method. This has been used intensively especially for tribological high-load contacts such as a vehicle brake. In Fig. 8, such a technical brake is shown.

Brakes show a highly dynamic friction coefficient. This is correlated with an extremely rich topographical and chemical dynamic in the boundary layer during the braking process.

Due to the complex material composition in brake pads (often more than 25 different materials), the friction process is supplied through a true a warehouse of materials in the boundary layer. Depending on the load and its duration, this process uses various chemical and physical processes which are technically designed to guarantee the quality and comfort of the brake pad. These processes are only understood to a small extend, and often appeared in a trial and error process to become the knowhow of a company. In addition to chemical processes, the boundary layer shows a characteristic topography. The creation of this topography can be explained with Fig. 9.

The main chemical structure of the brake material is a relatively soft polymer matrix with embedded small and very hard particles (e.g. $\mathrm{SiO}_{2}$ particles). All other components seem to only modify the process, which is described below and they will be ignored in this study [10]. A small section of the simplified coating with only one hard inhomogeneity is shown below.

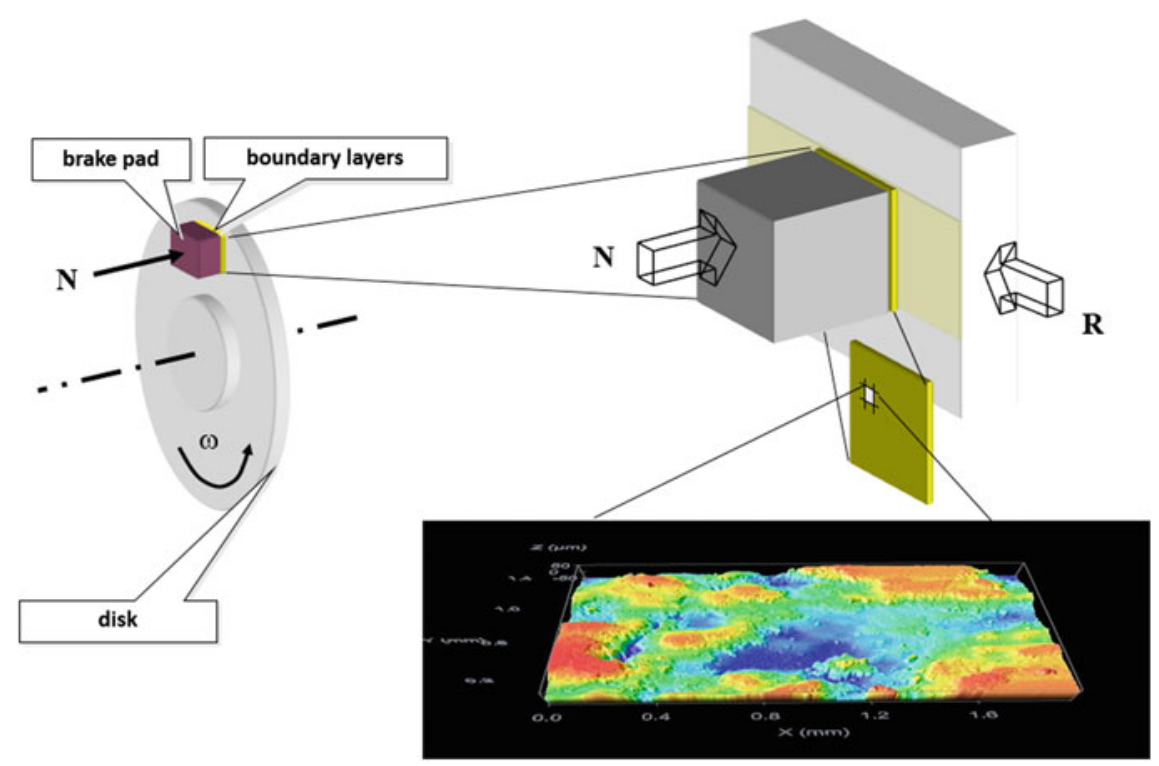

Fig. 8 Technical brake and presentation of the topography of the friction boundary layer 


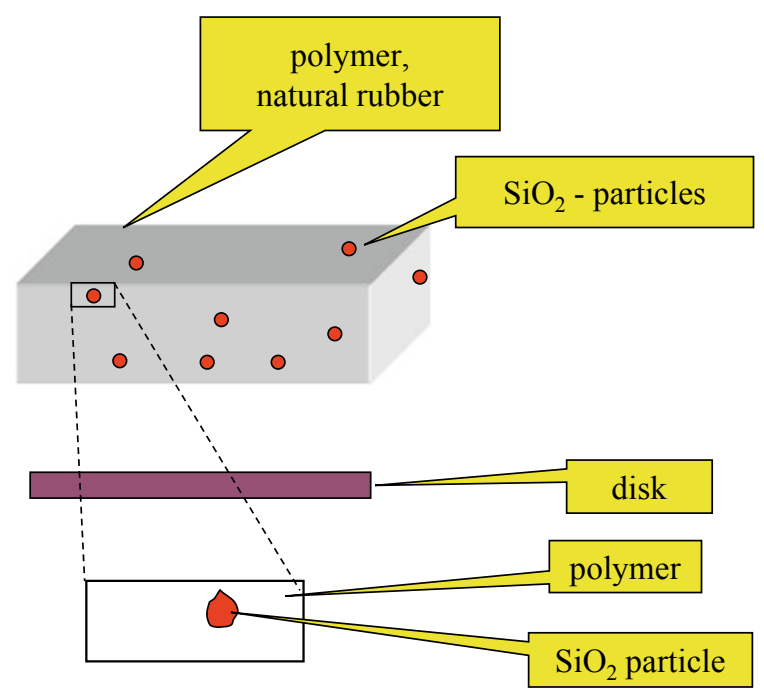

Fig. 9 Composition of a brake pad [3]

When the brake disc is pressed against the brake pad, abrasion occurs on the soft polymer matrix. The wear particles are transported along the contact zone. Some of the wear particles stay on the brake disc and after the disc has rotated, they return to the contact zone, while other particles are released into the environment [10].

This wear causes the hard particles to come to the surface of the pad. In the following, two modes may be observed. On the one hand, the flow of wear particles of the boundary layer is disturbed. On the other hand, the hard particles are pressed into the polymer matrix, as the soft matrix around the particle wears much more than the particle itself. As a result, an increase in the normal and tangential stress can be observed in the area of the hard particle. This results in an increase of the local temperature (see Fig. 10). The increase in stress and heat results in a process similar
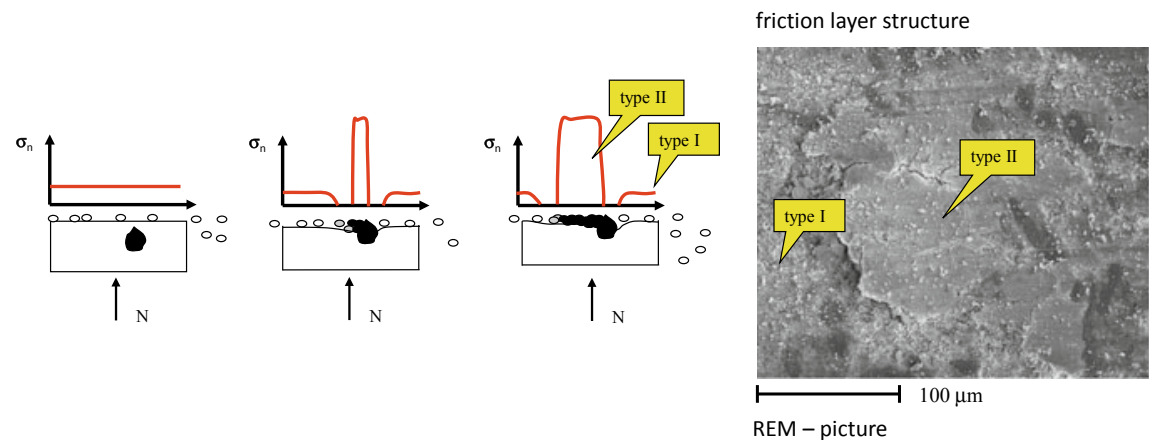

Fig. 10 Contact areas of the pad surface with normal stress distribution [10] 
$\mathrm{t}_{0}$

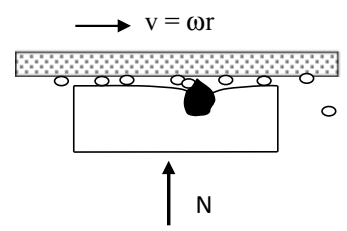

$\mathrm{t}_{1}$

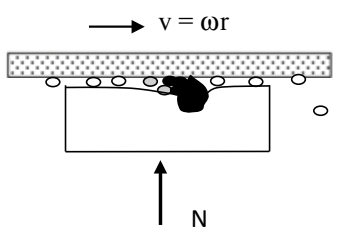

$t_{2}$

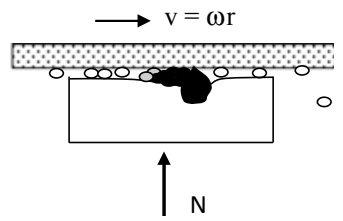

Fig. 11 Growth of contact patches [10]

to melting or sintering, whereby the wear particles together with the hard particle form an agglomeration modified by alloying processes and thin hard contact patches are formed (see Fig. 11) [10].

This process will divide the boundary layer surface into two contact areas. Type 1 is a contact area that consists of a rather soft polymer matrix that is relatively rough, heavily worn and has little frictional power. The second contact area is represented by the very hard and more wear-resistant patches (Type 2), which have a smooth surface and are mainly responsible for the main part of the friction power. They are ultimately responsible for the grey cast iron disc of the brake to wear and for iron entering the boundary layer chemistry.

These contact areas are dynamic. The patches grow due to friction power. However, their size is limited. If the total frictional power of a contact patch becomes too large, the patch cracks (see Fig. 12). The worn patch fragments represent an essential part of the wear particles of the braking process.

In the friction boundary layer, heat and wear are significant for the described dynamic process of topography change. Wear produces particles, and together with the heat the friction-intensive contact patches are born. Wear itself also ensures that the service life of these patches is limited. The total area of the patches is correlated with the current frictional power.

Friction is therefore a dynamic equilibrium. Not the friction coefficient itself, but its time derivative is defined by this process (see Fig. 13).

Dynamic friction laws can be derived from this idea [10]. Coulomb's friction law can be represented as the simplest dynamic equilibrium possible

$\mathrm{t}_{0}$

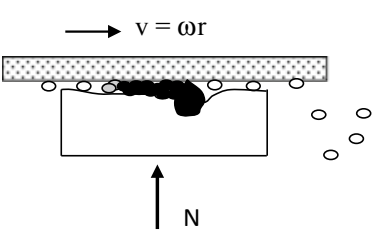

$t_{1}$

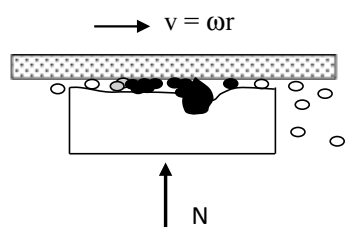

$t_{2}$

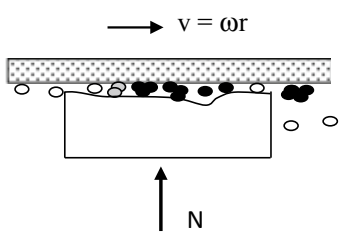

Fig. 12 Wear of contact patches [10] 


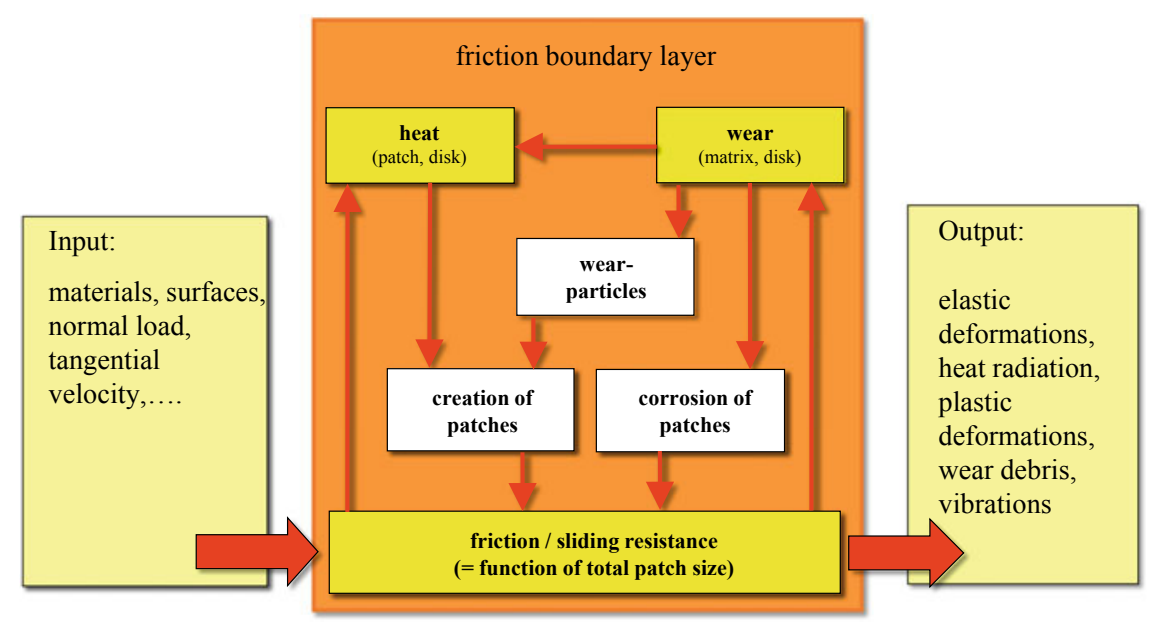

Fig. 13 Interactions in the boundary layer during the friction process [10]

$$
\dot{\mu}=0 \text {. }
$$

Volume-based Cellular Automata (CA) are a useful tool to simulate patch dynamics in the boundary layer (see Fig. 14). For the implementation, two areas are separated, as shown in Fig. 13. The relatively soft polymer matrix and the hard patches are discretized by cells with different normal elasticity.

In the first step, the normal stresses in the cells are calculated with a specified normal force. The contact forces in the patch areas are significantly higher. The
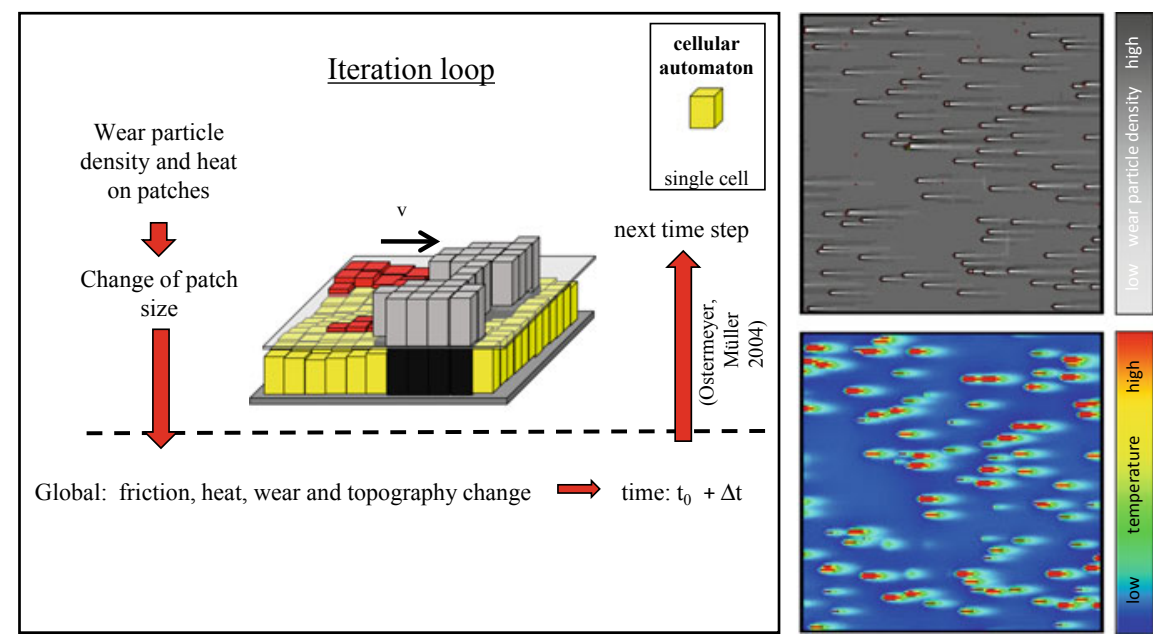

Fig. 14 Cellular automaton for the description of boundary layer dynamics 
tangential speed of the brake disc above the cells is also given as well as different friction coefficients on the polymer cells and contact patch cells.

This allows a wear volume or a wear particle density to be calculated in the contact area of the polymer matrix (see Fig. 14, right). Calculating the frictional power on the patch cells the temperature can be obtained on the respective patches. This temperature uses the density of the wear particles from the matrix to determine the growth of the patches. The total dissipation over time, the temperature level, and the size control the time of patch destruction.

This simulation model creates an iterative process that maps the patch dynamics and allows a statement to be made about global friction, heat, wear and topography changes. This allows the CA to discretize the local geometry of the brake pad, to introduce local material data, to map the local chemical status of the boundary layer and to formulate local balance equations. The balance equations serve to formulate the thermodynamic, physical and chemical rules of the CA [11, 12].

The simulation model confirmed the dynamics of the surface structure and the heterogeneous heat distribution of the pad. However, the simulation also shows that this boundary layer dynamics is a very general effect of a stable and robust selforganisation process on highly loaded friction surfaces. These simulations confirmed the structure of the transient friction coefficient of Ostermeyer in $[13,10]$, that the temperature $\mathrm{T}$ and the wear as a function of the friction power according to (2) are considered in the flow balance.

$$
\begin{aligned}
& \dot{\mu}=-\alpha\left(\left(\beta+\left|v_{r} \cdot N\right|\right) \cdot \mu-\gamma \cdot T_{p}\right) \\
& \dot{T}=-\delta\left(T_{p}-T_{0}-\varepsilon \cdot\left|v_{r} \cdot N\right|\right)
\end{aligned}
$$

\section{Natural Principles of Dissipation of Information}

Mesoscopic scales between the macroscopic and atomic world seem to be particularly suitable for observing locally resolved dynamics in the friction boundary layer. The core of these considerations, however, is the reduction of information that would theoretically make an exact calculation of friction on the atomic scale possible.

The problem with friction is that its characteristic dissipation on the macroscopic level does not exist on the atomic scale. It is neither the dissipation of energy nor the dissipation of material that is the core of friction, but the dissipation of information that should explain the macroscopic manifestations.

In search for general mechanisms of information reduction, one quickly finds that the mechanisms that are being used in physics are a natural way.

One of them is heat. Heat is a scalar field that collects all high-frequency effects on a molecular basis in a single parameter. Another mechanism that can be interpreted as dissipation of information is wear. Wear constantly changes the topography on an atomic scale. Therefore, it does not make sense to describe the geometric details at 
any time. Here, dynamic topography change rates as a scalar field of wear quantities make more sense.

Heat and wear are very good candidates to dissipate dynamic and geometric micro information, see Fig. 13, which is the basis for the dynamic friction models (7).

Certainly there are other elementary dissipation mechanisms of information which can be applied here in a useful way. Another mechanism is probably the uncertainty with which our experiences and measurements in the macroscopic world are recorded. A mathematically promising approach is offered by the Polymorphic Uncertainty Analysis.

Frictional systems can perform self-excited vibrations. During braking, they occur as an unpleasant noise, the so-called squealing. These self-excited states can be analyzed considering an eigenvalue problem. The uncertainty in the friction prediction induces an uncertainty in the stability limits of the model. Minimizing these uncertainties is necessary to enable more reliable brake designs. In Fig. 15 an uncertainty calculation has been carried out in the complex eigenvalue analysis of a simple brake model [14].

Here, Coulomb's friction law is compared to the dynamic friction law with respect to the confidence interval for stability limits in such an eigenvalue analysis. With the confidence interval, it is possible to quantify uncertainties that are present in the friction laws. If the parameters of Coulomb's friction law and the parameters of Ostermeyer's friction law are determined from concrete measured values, and the uncertainty of the friction coefficient is determined from these values, the model of the brake and the technique of complex eigenvalue analysis result in some uncertainty of the stability limits.

It is shown that the uncertainty interval for Coulomb's description is 15 times greater than for the dynamic friction law. This large difference is a consequence of the dynamic a-priori knowledge, which is represented in the flow balance conditions.

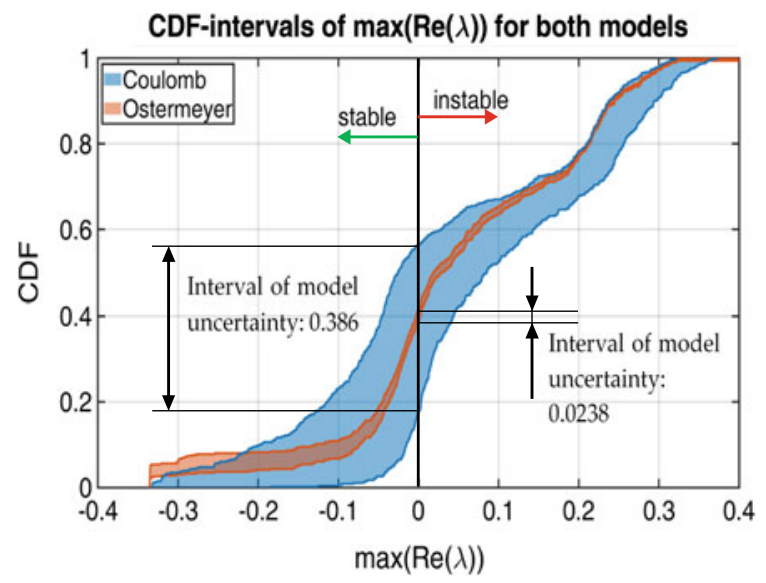

Fig. 15 Comparison of the Coulomb friction model and the Ostermeyer friction model with respect to polymorphic uncertainty and stability analysis [14] 


\section{Conclusion and Discussion}

Friction is a highly complex and dynamic phenomenon that is still not fully understood. One of the challenges in describing friction are the manifold interactions of dynamic effects on different time and length scales. Mesoscopic methods are an excellent tool to cope with the complexity of friction.

In this paper, two different approaches are discussed, which can describe the friction process in technical brakes with higher precision. One is specific particle methods, the other is volume-based cellular automata. Both methods use the mobility on the temporal and local scales between the atomic and macroscopic scale.

More general is the method of Movable Cellular Automata, which combines the advantages of the particle world with the advantages of the world of Cellular Automata. The creator of this method, Sergey Psakhie was a visionary. His methods have found the way not only into friction, but into many other areas [15].

\section{References}

1. Ostermeyer G-P (2003) Thesen zur Modellierung von Reibung und Verschleiß. Tribol Schmierungstech 4:18-22

2. Coulomb CA (1785) Die Theorie einfacher Schwingungen. Memoires de mathematique et de physique de l'Academie des Sciences 10:161-331

3. Ostermeyer G-P (2010) Dynamic friction laws and their impact on friction induced vibrations. SAE technical paper 2010-01-1717, pp 1-27

4. Ostermeyer G-P (1999) A mesoscopic particle method for description of thermomechanical and friction processes. Phys Mesomech 6:25-32

5. Ostermeyer G-P (2007) The mesoscopic particle approach. Tribol Int 40(6):953-959

6. Boltzmann L (1897) Vorlesungen über die Prinzipe der Mechanik. Verlag von Johann Ambrosius Barth, Leipzig

7. Greenspan D (1972) A discrete numerical approach to fluid dynamics. Inf Process 71:12971304

8. Greenspan D (1988) Particle modeling in science and technology. In: Numerical methods, Proceedings 4th conference, Miskolc/Hung, 1986, Colloquia Mathematica Societatis Janos Bolyai 50, pp 51-66

9. Ostermeyer G-P (1996) Many particle systems. Dynamical problems in mechanical systems. In: Proceedings of the 4th Polish-German workshop, July 30-Aug 05, 1995, Polska Akademia Nauk, IPPT Warszawa, Warsaw (PL), pp 249-259

10. Ostermeyer G-P (2003) On the dynamics of the friction coefficient. Wear 254(9):852-858

11. Ostermeyer G-P, Müller M (2005) Dynamic Interaction of friction and surface topography in brake systems. Tribol Int 39(5):370-380

12. Ostermeyer G-P, Müller M (2008) New insights into the tribology of brake systems. Proc Inst Mech Eng Part D J Autom Eng 222(7):1167-1200

13. Ostermeyer G-P (2001) Friction and wear of brake systems. Forsch Ingenieurwes 66:267-272

14. Ostermeyer G-P, Müller M, Brumme S, Srisupattarawanit T (2019) Stability analysis with an $\mathrm{NVH}$ minimal model for brakes under consideration of polymorphic uncertainty of friction. Vibration 2:135-156

15. Psakhie SG, Horie Y, Ostermeyer G-P, Smolin AYu, Korostelev SYu, Shilko EV, Dmitriev AI, Blatnik S, Špegel M, Zavšek S (2001) Movable cellular automata method for simulating materials with mesostructured. Theoret Appl Fract Mech 37(1-3):311-334 
Open Access This chapter is licensed under the terms of the Creative Commons Attribution 4.0 International License (http://creativecommons.org/licenses/by/4.0/), which permits use, sharing, adaptation, distribution and reproduction in any medium or format, as long as you give appropriate credit to the original author(s) and the source, provide a link to the Creative Commons license and indicate if changes were made.

The images or other third party material in this chapter are included in the chapter's Creative Commons license, unless indicated otherwise in a credit line to the material. If material is not included in the chapter's Creative Commons license and your intended use is not permitted by statutory regulation or exceeds the permitted use, you will need to obtain permission directly from the copyright holder.

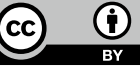

